\title{
ATP turnover and glucose dependency in hematopoietic stem/ progenitor cells are increased by proliferation and differentiation
}

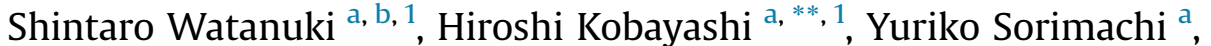 \\ Masamichi Yamamoto ${ }^{c}$, Shinichiro Okamoto ${ }^{b}$, Keiyo Takubo ${ }^{\text {a, * }}$ \\ a Department of Stem Cell Biology, Research Institute, National Center for Global Health and Medicine, 1-21-1, Toyama, Shinjuku-ku, Tokyo 162-8655, Japan \\ b Division of Hematology, Department of Medicine, Keio University School of Medicine, Tokyo 160-8582, Japan \\ c Department of Nephrology, Kyoto University Graduate School of Medicine, Kyoto 606-8507, Japan
}

\section{A R T I C L E I N F O}

\section{Article history:}

Received 3 April 2019

Received in revised form

16 April 2019

Accepted 17 April 2019

Available online 25 April 2019

\section{Keywords:}

Hematopoietic stem cell

Granulocyte/macrophage progenitor

Stem cell metabolism

Adenosine triphosphate

\begin{abstract}
A B S T R A C T
Hematopoietic stem cells (HSCs) are quiescent cells in the bone marrow niche and are relatively dependent on glycolytic ATP production. On the other hand, differentiated cells, including hematopoietic progenitor cells (HPCs), preferentially generate ATP via oxidative phosphorylation. However, it is unclear how cellular differentiation and the cell cycle status affect nutritional requirements and ATP production in HSCs and HPCs. Using a newly developed culture system, we demonstrated that survival of HPCs was strongly dependent on glucose, whereas quiescent HSCs survived for a certain duration without glucose. Among HPCs, granulocyte/monocyte progenitors (GMPs) were particularly dependent on glucose during proliferation. By monitoring the ATP concentration in live cells, we demonstrated that the ATP level was maintained for a short duration without glucose in HSCs, possibly due to their metabolic flexibility. In addition, HSCs exhibited low ATP turnover, whereas HPCs including GMPs demonstrated high ATP turnover and required efficient ATP production from glucose. These findings show that ATP turnover and nutritional requirements differ between HSCs and HPCs according to the cell cycle and differentiation status.
\end{abstract}

๑) 2019 Elsevier Inc. All rights reserved.

\section{Introduction}

Hematopoiesis in adult mammals is maintained by both actively cycling hematopoietic progenitor cells (HPCs) and quiescent hematopoietic stem cells (HSCs), both of which contribute to production of all hematopoietic lineages [1]. HSCs are metabolically inactive and relatively maintained via glycolytic ATP production,

Abbreviations: CLP, common lymphoid progenitor; ECAR, extracellular acidification rate; Epo, erythropoietin; FCS, fetal calf serum; FRET, Förster resonance energy transfer; GFP, green fluorescent protein; GMP, granulocyte/macrophage progenitor; HPC, hematopoietic progenitor cell; HSC, hematopoietic stem cell; HSPC, hematopoietic stem/progenitor cell; IL, interleukin; MEP, megakaryocyte/ erythroid progenitor; MFI, mean fluorescence intensity; OCR, oxygen consumption rate; OXPHOS, oxidative phosphorylation; SCF, stem cell factor; TPO thrombopoietin.

* Corresponding author.

** Corresponding author.

E-mail addresses: hikobayashi-tky@umin.ac.jp (H. Kobayashi), keiyot@gmail. com (K. Takubo)

1 These authors contributed equally to this study. while multipotent and lineage-committed progenitors, hereafter called HPCs, depend more on oxidative phosphorylation (OXPHOS) in mitochondria for ATP production [2,3]. Under stressed conditions, HSCs, similar to HPCs, actively proliferate, shift their metabolic program to OXPHOS-dependent ATP production, and activate a metabolic shunt of glycolysis, the pentose phosphate pathway, and purine biosynthesis [4,5]. Production and consumption (i.e., turnover) of ATP are strictly controlled in a cooperative manner to maintain the ATP concentration in living cells. For example, phosphofructokinase, the rate-limiting enzyme of glycolysis, is allosterically inhibited by ATP, while its enzymatic activity is enhanced by ADP [6]. In addition, the activities of enzymes related to the tricarboxylic acid cycle, including pyruvate dehydrogenase [7], isocitrate dehydrogenase, and $\alpha$-ketoglutarate dehydrogenase [8], differ according to the ATP concentration.

Glucose is catabolized to produce ATP via glycolysis or OXPHOS in living cells. Hematopoietic stem/progenitor cells (HSPCs) respond to various external stresses such as infection, bleeding, and radiation. Thus, both extrinsic cues such as cytokines and the intrinsic status (cell cycle and/or differentiation) may affect glucose 
and ATP metabolism in HSPCs. However, it is unclear whether alterations in glucose metabolism in response to changes in cytokine concentrations differ among HSPC fractions. Here, we recapitulated steady-state and proliferative conditions by changing cytokine concentrations and determined the glucose demand of each HSPC fraction. In addition, by measuring the ATP concentration in live hematopoietic cells under the defined culture conditions, we revealed that HSCs flexibly alter their glucose demand for energy production in response to cytokines, while HPCs invariably require glucose in all environmental conditions tested.

\section{Materials and methods}

\subsection{Animals}

C57BL/6J mice (SLC Japan) aged 10-15 weeks were used in all experiments, unless otherwise stated. ATP-biosensor (GOATeam2)-knock-in mice were previously described [paper in preparation]. Animal experiments were approved by the Institutional Review Board of the National Center for Global Health and Medicine. Both male and female mice were used in experiments.

\subsection{Antibodies for flow cytometry}

HSCs of C57BL/6J mice were stained with anti-lineage marker (CD4, CD8a, Gr-1, Mac-1, Ter-119, B220)-PerCP-Cy5.5, anti-c-KitAPC-Cy7, anti-Sca-1-PE-Cy7, anti-CD150-PE, anti-CD48-FITC, and anti-Flt3-APC antibodies. HSCs of GO-ATeam2-knock-in mice were stained with anti-lineage marker-PerCP-Cy5.5, anti-c-Kit-APC-Cy7, anti-Sca-1-PE-Cy7, anti-CD150-BV421, and anti-CD48-APC antibodies. HSPCs of C57BL/6J mice were stained with anti-lineage marker-PerCP-Cy5.5, anti-c-Kit-APC-Cy7, anti-Sca-1-PE-Cy7, antiIL-7R-PE, anti-CD34-FITC, anti-Flt3-APC, and anti-CD16/32-Alexa Fluor 700 antibodies. HSPCs of GO-ATeam2-knock-in mice were stained with anti-lineage marker-PerCP-Cy5.5, anti-c-Kit-APC-Cy7, anti-Sca-1-PE-Cy7, anti-CD34-BV421, and anti-CD16/32-Alexa Fluor 700 antibodies. All antibodies were purchased from BD Biosciences, BioLegend, TONBO Biosciences, or eBioscience.

\subsection{Flow cytometric analysis and isolation of hematopoietic cells}

Murine bone marrow cells were collected from two femurs and tibias for analysis and isolation of HSCs and HPCs. Following hemolysis, cells were incubated with CD117 MicroBeads, mouse (Miltenyi). c-Kit ${ }^{+}$cells were collected using AutoMACS (Miltenyi), and then surface markers were stained with fluorophoreconjugated antibodies. HSCs and HPCs were phenotypically defined based on the presence and absence of surface markers as follows: HSCs, lineage ${ }^{-} \mathrm{Sca}-{ }^{+}{ }^{+} \mathrm{c}-\mathrm{Kit}^{+} \mathrm{Flt3}{ }^{-} \mathrm{CD} 150^{+} \mathrm{CD} 48^{-}$; MPP4 cells, lineage ${ }^{-} \mathrm{Sca}-1^{+} \mathrm{c}-\mathrm{Kit}^{+} \mathrm{Flt} 3^{+}$; common lymphoid progenitors (CLPs), lineage ${ }^{-} \mathrm{Sca}-1^{\text {low }} \mathrm{c}-\mathrm{Kit}^{\text {low }} \mathrm{IL}_{-}-7 \mathrm{R}^{+} \mathrm{Flt} 3^{+}$(for cell culture) or lineage ${ }^{-} \mathrm{Sca}-1^{\text {low }} \mathrm{C}-\mathrm{Kit}^{\text {low }}{ }^{\mathrm{IL}-7 \mathrm{R}^{+}}$(for flux analyzer); megakaryocyte/ erythroid progenitors (MEPs), lineage ${ }^{-}$Sca- $1^{-}$c-Kit ${ }^{+}$CD16/ $32^{-} \mathrm{CD} 34^{-}$; and granulocyte/macrophage progenitors (GMPs), lineage ${ }^{-} \mathrm{Sca}-1^{-} \mathrm{c}-\mathrm{Kit}^{+} \mathrm{CD} 16 / 32^{+} \mathrm{CD} 34^{+}$. The methods used to identify and isolate HSCs and other hematopoietic cells were described previously [9-11]. Cell sorting was performed using a FACS Aria II or FACS Aria IIIu instrument (BD Biosciences). Data were analyzed with FlowJo software (Treestar).

For analysis of cultured cells, most $(170 \mu \mathrm{L})$ of the medium in each well of a 96-well plate was aspirated and samples were stained with $10 \mu \mathrm{L}$ of an antibody cocktail for $30 \mathrm{~min}$ at $4{ }^{\circ} \mathrm{C}$. HSCs were stained with anti-lineage marker (CD4, CD8a, Gr-1, Mac-1, B220, Ter-119)-PerCP-Cy5.5, anti-c-Kit-APC-Cy7, anti-Sca-1-PE-Cy7, anti-CD150-PE, anti-CD48-FITC, and anti-CD41-APC antibodies.
HSPCs were stained with anti-c-Kit-APC-Cy7, anti-Gr-1-PE-Cy7, anti-Mac-1-PE-Cy7, anti-B220-PE, anti-Ter119-FITC, and anti-CD41APC antibodies. In all cases, $0.1 \mu \mathrm{L}$ of each antibody was added to each well. After incubation, $100 \mu \mathrm{L}$ of phosphate-buffered saline containing $2 \%$ fetal calf serum (FCS) was added to each well, and the plates were centrifuged for $5 \mathrm{~min}$ at $4{ }^{\circ} \mathrm{C}$. Thereafter, $100 \mu \mathrm{L}$ of the supernatant was aspirated and the cell pellet was resuspended in $200 \mu \mathrm{L}$ of phosphate-buffered saline containing $2 \%$ FCS and $0.1 \%$ propidium iodide (Thermo Fisher Scientific) as well as 0.25\% FlowCheck Fluorospheres (Beckman Coulter). Samples were acquired in fast mode by an autosampler-equipped MACSQuant analyzer (Miltenyi), and volumes of $100 \mu \mathrm{L}$ were analyzed. Data were exported as FCS files and analyzed using Flowjo software. The absolute cell number was estimated by the bead count of Flow-Check $(\sim 1000 / \mu \mathrm{L})$.

\subsection{Cell culture}

The basal culture medium of isolated cells was RPMI1640 (Nacalai Tesque). The medium was supplemented with $4 \%$ bovine serum albumin (Sigma-Aldrich), insulin ( $2 \mathrm{mg} / \mathrm{L})$, transferrin $(1.1 \mathrm{mg} / \mathrm{L})$, sodium selenite $(0.0134 \mathrm{mg} / \mathrm{L}), 2$ 2-mercaptoethanol $(55 \mu \mathrm{M})$, hypoxanthine $(2.1 \mathrm{mg} / \mathrm{L})$, and thymidine $(0.37 \mathrm{mg} / \mathrm{L})$. Glucose $(3151 \mathrm{mg} / \mathrm{L})$ was added when indicated. Cells were cultured in $200 \mu \mathrm{L}$ of medium in 96 -well U-bottom plates in an atmosphere containing $1 \% \mathrm{O}_{2}$ and $5 \% \mathrm{CO}_{2}$ at $37^{\circ} \mathrm{C}$.

\subsection{Cytokine treatments}

For HSCs, $3 \mathrm{ng} / \mathrm{mL}$ murine recombinant stem cell factor (SCF) and $0.1 \mathrm{ng} / \mathrm{mL}$ human recombinant thrombopoietin (TPO) were added to quiescence-maintaining cultures, while $1 \mathrm{ng} / \mathrm{mL}$ SCF and $100 \mathrm{ng} / \mathrm{mL}$ TPO were added to proliferative cultures. For HSPCs, murine SCF, murine Flt3 ligand, murine interleukin (IL)-3, murine IL-6, murine IL-7, and human erythropoietin (Epo) were added at the indicated concentrations. All cytokines were purchased from Peprotech.

\subsection{Flux analyzer}

The extracellular acidification rate (ECAR, a surrogate for glycolysis) and oxygen consumption rate (OCR, to assess mitochondrial respiration) were measured using a Seahorse XF96 extracellular flux analyzer according to the manufacturer's instructions (Agilent Technologies). Briefly, sorted cells were transferred to a culture plate pre-coated with Cell-Tak (Corning) and containing pre-warmed SF-O3 medium lacking supplements (HEPES, insulin, transferrin, ethanolamine, sodium selenite, and sodium bicarbonate), and centrifuged at $200 \times \mathrm{g}$ for $5 \mathrm{~min}$. The OCR and ECAR were measured at baseline and after sequential addition of the following respiratory inhibitors at the specified final concentrations: $1 \mu \mathrm{M}$ oligomycin (an inhibitor of ATP synthase), $2 \mu \mathrm{M}$ FCCP (an uncoupling agent of mitochondrial respiration), $0.5 \mu \mathrm{M}$ rotenone/antimycin (an inhibitor of mitochondrial complex I/III), and $50 \mathrm{mM}$ 2-deoxy-D-glucose (an inhibitor of glycolysis).

\section{7. cDNA synthesis and quantitative RT-PCR}

RNA was extracted from sorted HSCs and GMPs using an RNeasy mini kit (QIAGEN). cDNA was synthesized using SuperScript VILO (Thermo Fisher Scientific) in a final volume of $20 \mu \mathrm{L}$ according to the manufacturer's instructions. qPCR was performed using SYBR Premix ExTaqT⿳ ${ }^{\mathrm{TM}}$ Ila (TaKaRa Bio) according to the manufacturer's instructions. A mixture containing $45 \mu \mathrm{L}$ of SYBR Premix, $0.36 \mu \mathrm{L}$ each of forward and reverse primers, $1.8 \mu \mathrm{L}$ of Rox II dye, $41.48 \mu \mathrm{L}$ of 
distilled water, and $1 \mu \mathrm{L}$ of cDNA solution was generated, and $20 \mu \mathrm{L}$ was dispensed to each of four wells of an assay plate. PCR analysis was performed using an ABI 7500 Fast Real-Time PCR System (Applied Biosystems) under the following conditions: $95^{\circ} \mathrm{C}$ for $10 \mathrm{~s}$ followed by 40 cycles of $95^{\circ} \mathrm{C}$ for $5 \mathrm{~s}$ and $60^{\circ} \mathrm{C}$ for $34 \mathrm{~s}$. Expression levels were determined as $2^{\wedge}$ ( $\mathrm{Ct}$ value - mean $\mathrm{Ct}$ value of $\beta$-actin) and normalized against those in control samples, unless otherwise stated.

\subsection{Förster resonance energy transfer (FRET) value measurement}

FRET values were determined by calculating the ratio between the mean fluorescence intensities (MFIs) of Kusabira Orange and green fluorescent protein (GFP) when excited by light of $488 \mathrm{~nm}$ wavelength. MFIs were analyzed with a FACS Aria II or FACS Aria IIIu instrument (BD Biosciences). FCS files were converted to CSV files on a public server of Gene Pattern (Broad Institute), and MFIs were extracted. FRET values were calculated based on the MFIs of GFP and Kusabira Orange using the following equation:

FRET value $=$ MFI of Kusabira Orange/MFI of GFP.

\subsection{Microarray data analysis}

cDNA microarray data for murine HSCs and HPCs were obtained from Gene Expression Omnibus (Accession number: GSE55525), and the indicated cell types were subjected to hierarchical clustering. Following removal of probes with low intensities (value $<8$ ) and $z$-score normalization for each probe, clustering was performed using the heatmap.2 function of the gplots package. The gene sets KEGG_GLYCOLYSIS_GLUCONEOGENESIS and KEGG_OXIDATIVE_PHOSPHORYLATION were obtained from mSigDB of the gsea website (http://software.broadinstitute.org/gsea/msigdb/). Ldhd, G6pdx, Pfkfb1, Pfkfb2, Pfkfb3, and Pfkfb4 were added to the glycolysis set. Microarray probes with values less than 8 were removed from the analysis.

\subsection{Statistical analysis}

Two groups were compared using the unpaired two-tailed Student's t-test. Data are shown as means \pm standard deviation. $p$ values less than 0.05 (indicated by asterisks in the figures) were considered statistically significant.

\section{Results}

\subsection{HPCs and proliferating HSCs constantly require glucose for survival ex vivo, while quiescent HSCs do not}

To determine the metabolic characteristics of HSCs and HPCs at steady state and during proliferation, we utilized ex vivo culture conditions that maintained quiescence or induced proliferation. We previously established these culture conditions for HSCs [12]; therefore, we first determined the minimal cytokine combinations and concentrations that maintained quiescence (quiescent conditions) of HPCs. Culture in the presence of increased concentrations of the minimal cytokine combinations was used to induce proliferation (proliferative conditions) of HPCs. HSCs and HPCs were cultured in these conditions for 6 and 4 days, respectively (Fig. 1A and see Methods). The number of HSCs was unaffected by culture in quiescent conditions in the presence or absence of glucose, but was decreased by culture in proliferative conditions in the absence of glucose (Fig. 1B and C). By contrast, MPP4 cells, GMPs, MEPs, and CLPs did not survive upon culture in the absence of glucose, regardless of the concentrations of cytokines (Fig. 1D-G). Other than HSCs, only GMPs proliferated and differentiated upon culture in the presence of a low SCF concentration $(<3 \mathrm{ng} / \mathrm{mL})$, which is consistent with a recent report that GMPs require less nichederived SCF than MEPs [13]. Even under this condition, survival of GMPs was strongly dependent on the presence of glucose, in contrast with HSCs (Fig. 1G). Collectively, quiescent HSCs adapted to a low non-physiological glucose concentration for survival, while HPCs continuously required glucose irrespective of their cell cycle status. Thus, HPCs, including GMPs, constantly demand glucose.

\subsection{Glycolytic activity and oxygen consumption are highest in GMPs among HSPCS}

To further compare metabolism between HSCs and HPCs, the OCR and ECAR were calculated using an extracellular flux analyzer system. The baseline OCR, representing steady-state OXPHOS, was high in myeloid progenitors such as GMPs and MEPs. The maximal OCR after FCCP application was highest in GMPs (Fig. 2A). The ECAR, representing glycolytic activity, was constantly higher in GMPs than in other HSPCs (Fig. 2B). The OCR and ECAR were lower in CMPs than in GMPs and MEPs (Fig. 2A-D). Glycolytic activity of LSK cells was high relative to their OXPHOS activity (Fig. 2B, D, E), consistent with previous reports that undifferentiated quiescent hematopoietic cells are relatively dependent on glycolysis [14,15]. Among HSPCs, the OCR and ECAR were lowest in CLPs (Fig. 2A and $B)$. In summary, each HSPC fraction has unique characteristics in terms of their OCR and ECAR, and GMPs exhibit increased glycolysis and OXPHOS activity (and possibly a high ATP production ability).

\subsection{Expression of glycolytic/OXPHOS enzymes differs among HSPCS}

The cellular concentration of ATP is determined by its turnover (i.e., balance between the production and consumption rates) [16]. To determine the ATP production ability of HSPCs, we examined the expression levels of glycolytic/OXPHOS enzymes by qPCR and by analyzing cDNA microarray data available in a public database [17]. Among glycolytic enzymes, expression of Ldha and Pgam1, but not of Ldhb, was higher in GMPs than in HSCs (Fig. 2F), suggesting the robust preference for glycolytic energy production in GMPs. Consistent with glycolytic activity measured by a flux analyzer (Fig. 2B), expression of glycolytic/OXPHOS genes was high in GMPs, but low in HSCs, MPP cells, and CLPs (Fig. 2G). These results suggest that the expression levels of glycolytic/OXPHOS genes are dynamically regulated according to the differentiation stage.

\subsection{The ATP concentration is lowest in GMPs among HSPCS}

The ATP concentration reflects metabolic activity in cells. We utilized ATP-biosensor (GO-ATeam2)-knock-in mice to directly measure the intracellular ATP concentration in HSPCs [18]. The $\varepsilon$-subunit of bacterial ATP synthase is linked with the fluorescent proteins GFP and Kusabira Orange in these mice. FRET occurs upon ATP binding, thereby enabling quantification of the cellular ATP concentration (Fig. 3A and B).

Using these mice, FRET values in live hematopoietic cells were measured to determine the ATP concentration in HSPCs. Although the total amount of ATP is reportedly lower in HSCs than in progenitors, the ATP concentration was significantly higher in HSCs than in GMPs (Fig. 3C). HSCs, CMPs and MEPs showed identical levels of the ATP concentration. To confirm the relationship between the cell cycle status and ATP concentration, we cultured GOATeam2 knock-in HSCs and GMPs in the presence of high or low cytokine concentrations (Fig. 3D). FRET values were significantly lower when HSCs and GMPs were cultured in the presence of high cytokine concentrations than when they were cultured in the presence of low cytokine concentrations (Fig. 3E). In addition, FRET 
A

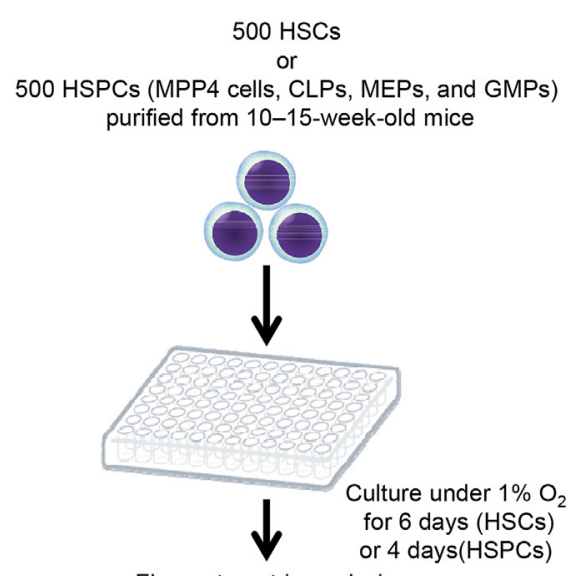

Flow cytometric analysis
B

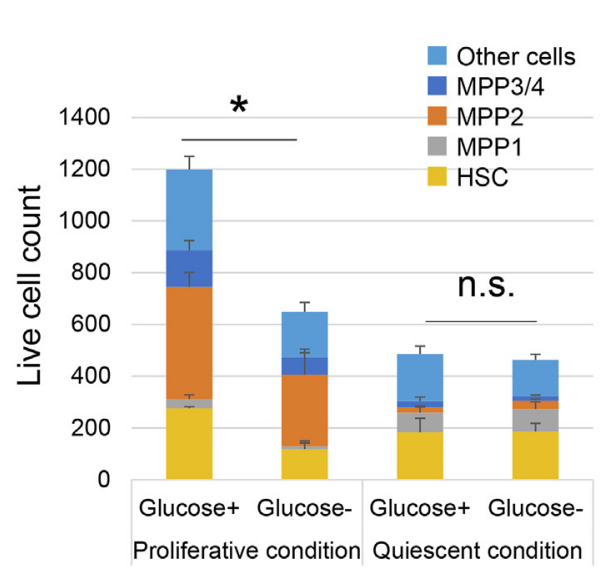

C

HSC culture

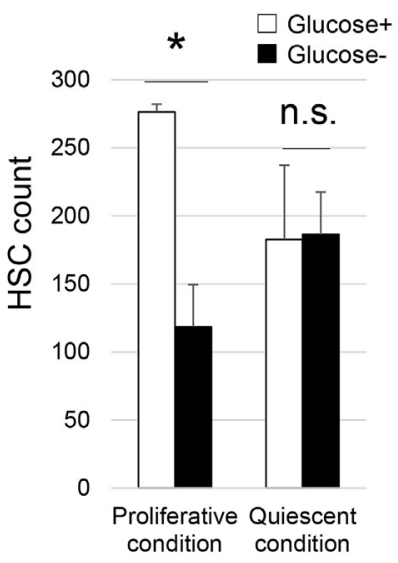

D

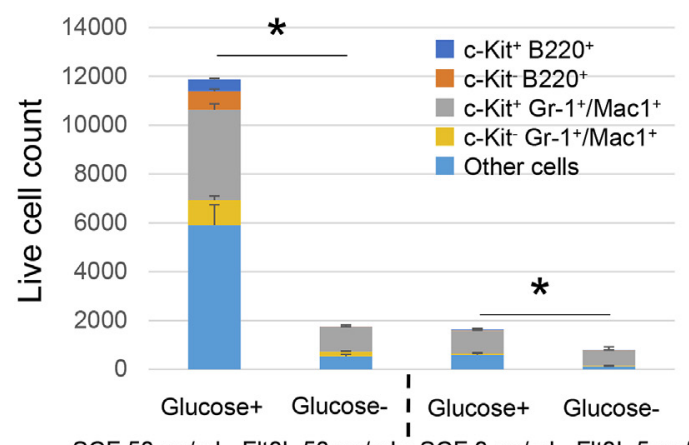

SCF $50 \mathrm{ng} / \mathrm{mL}$, Flt $3 \mathrm{~L} 50 \mathrm{ng} / \mathrm{mL}$ I SCF $3 \mathrm{ng} / \mathrm{mL}$, Flt $3 \mathrm{~L} 5 \mathrm{ng} / \mathrm{mL}$ IL-7 $100 \mathrm{ng} / \mathrm{mL}$, IL-6 $0.1 \mathrm{ng} / \mathrm{mLI} \mid \mathrm{LL}-710 \mathrm{ng} / \mathrm{mL}$, IL-6 $0.1 \mathrm{ng} / \mathrm{mL}$

\section{$\mathbf{F}$}

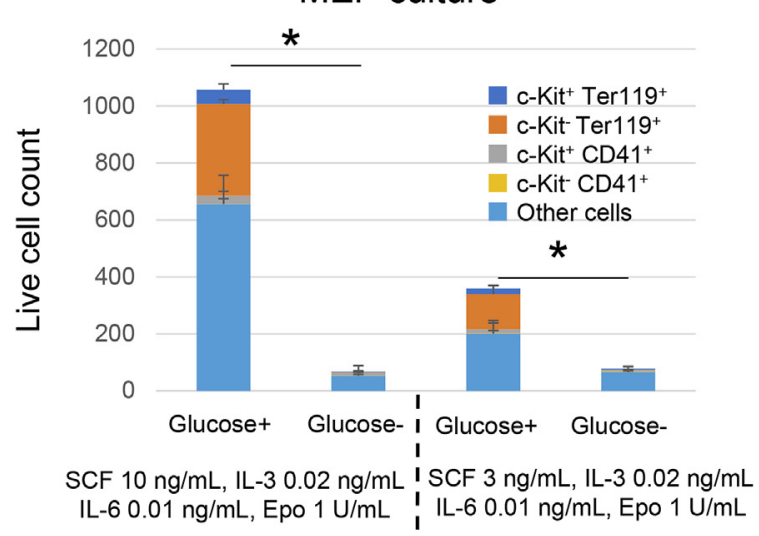

E

\section{CLP culture}

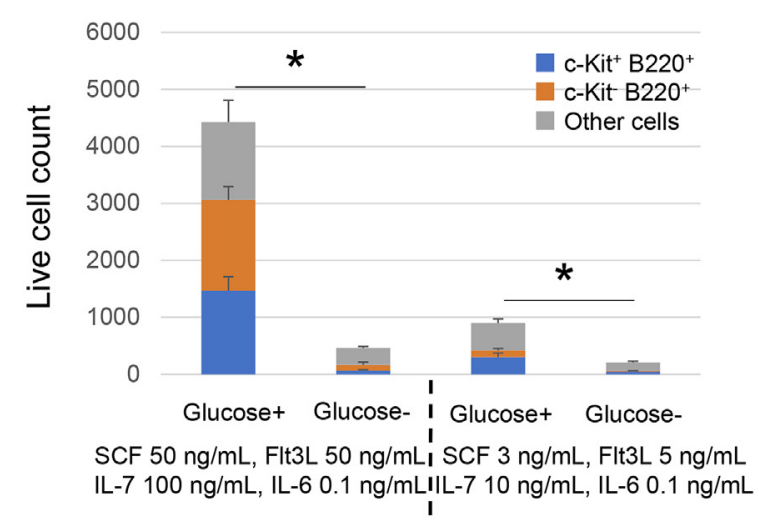

G

\section{GMP culture}

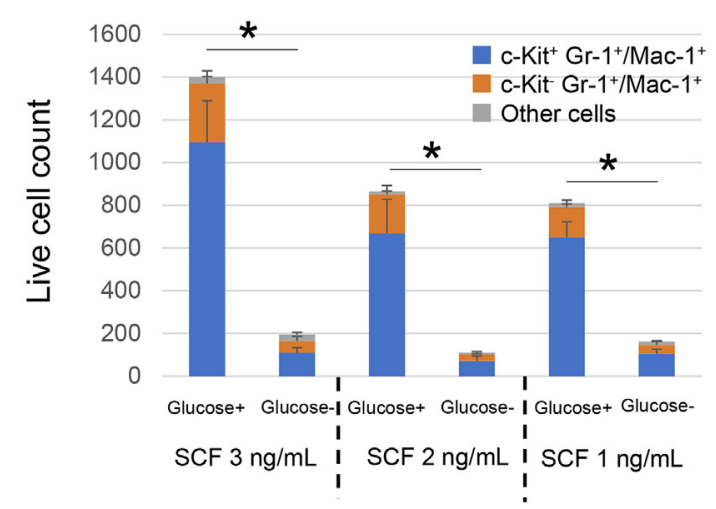

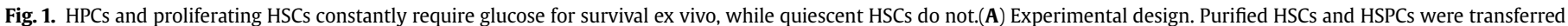

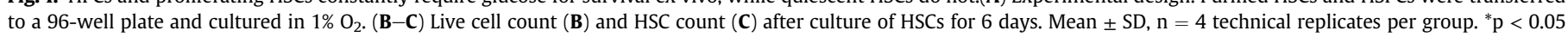

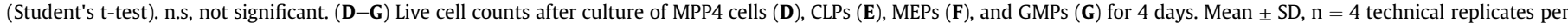
group. *p $<0.05$ (Student's t-test).

values were significantly lower in GMPs than in HSCs upon culture in the presence of high or low cytokine concentrations (Fig. 3E). These results suggest that the cellular ATP concentration differs among HPSCs and that proliferation and differentiation increase ATP consumption and reduce the ATP concentration in these cells.

\section{Discussion}

In this study, we demonstrated that HSCs required glucose in a context-dependent manner (Fig. 4A and B), whereas survival of HPCs was highly dependent on glucose at all cytokine 

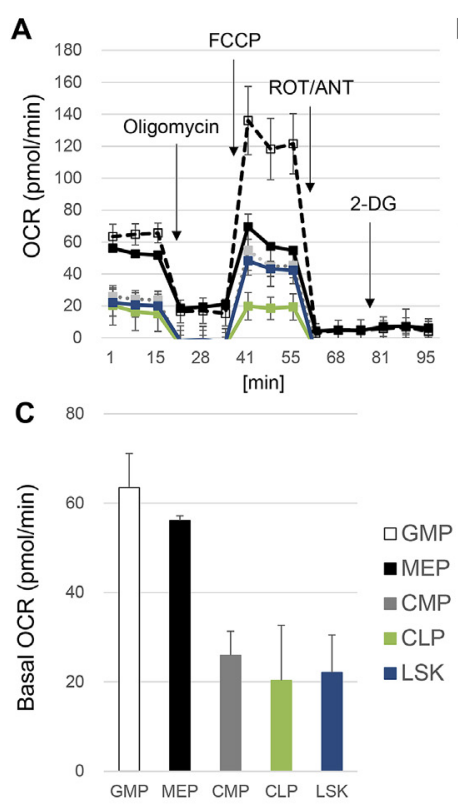

E Baseline OCR/ECAR

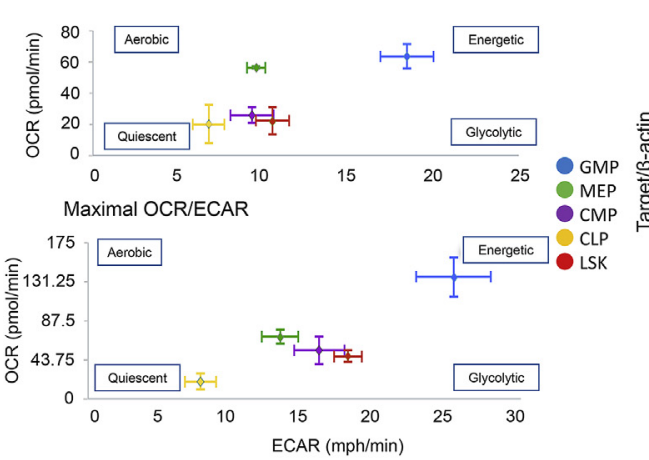

G

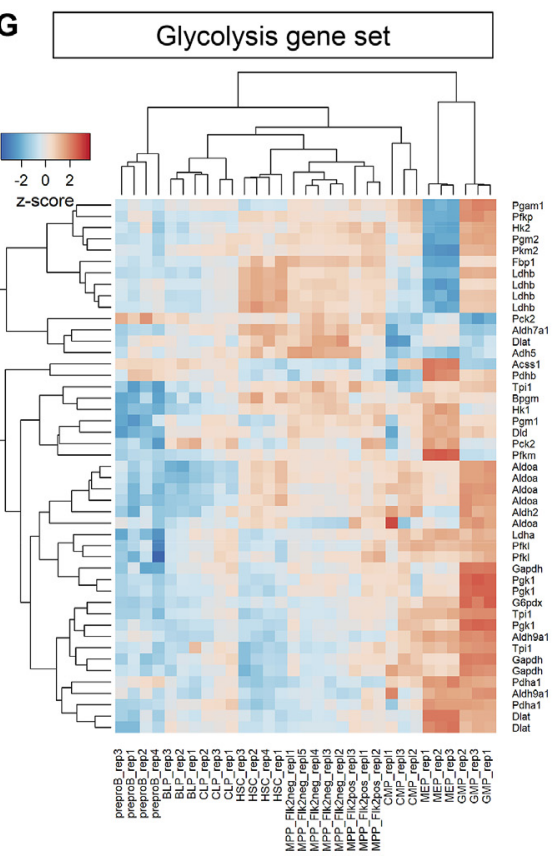

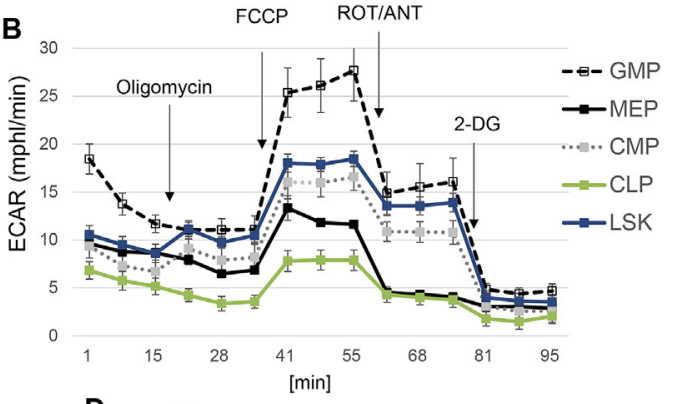

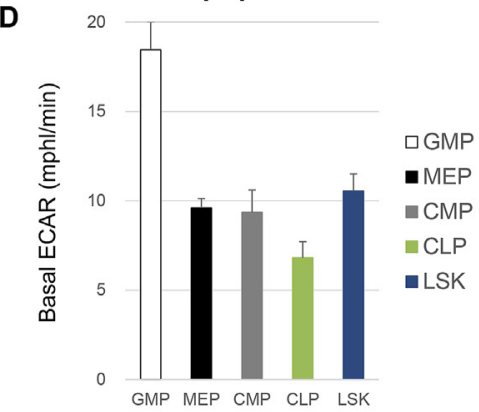

$\mathbf{F}$

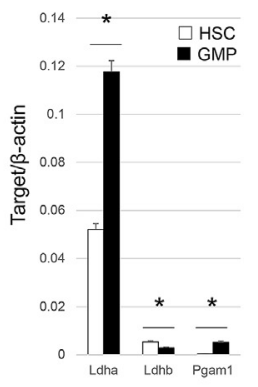

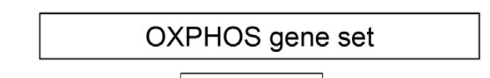

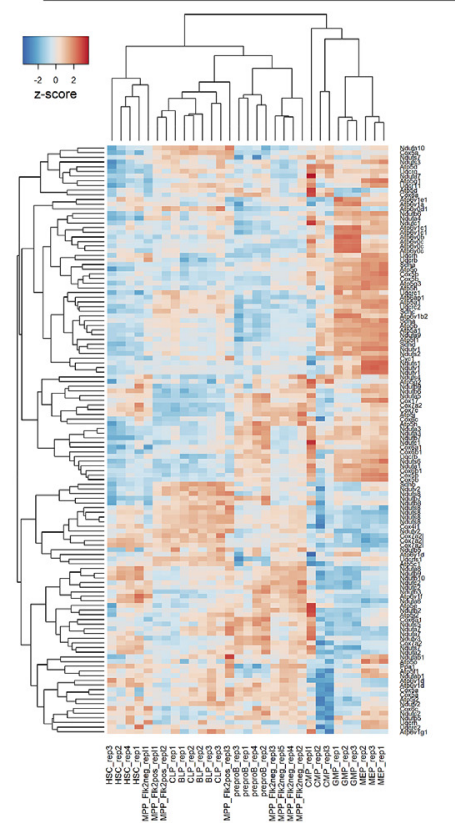

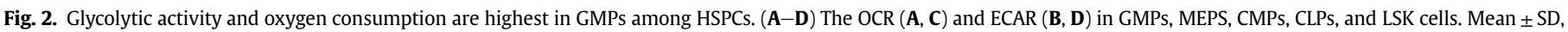

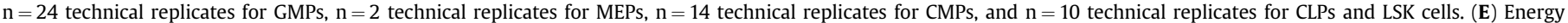

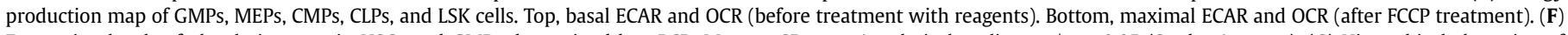

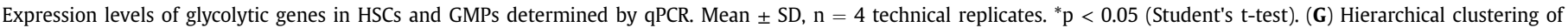
microarray data of HSPC fractions. Left, glycolysis gene set. Right, OXPHOS gene set. 

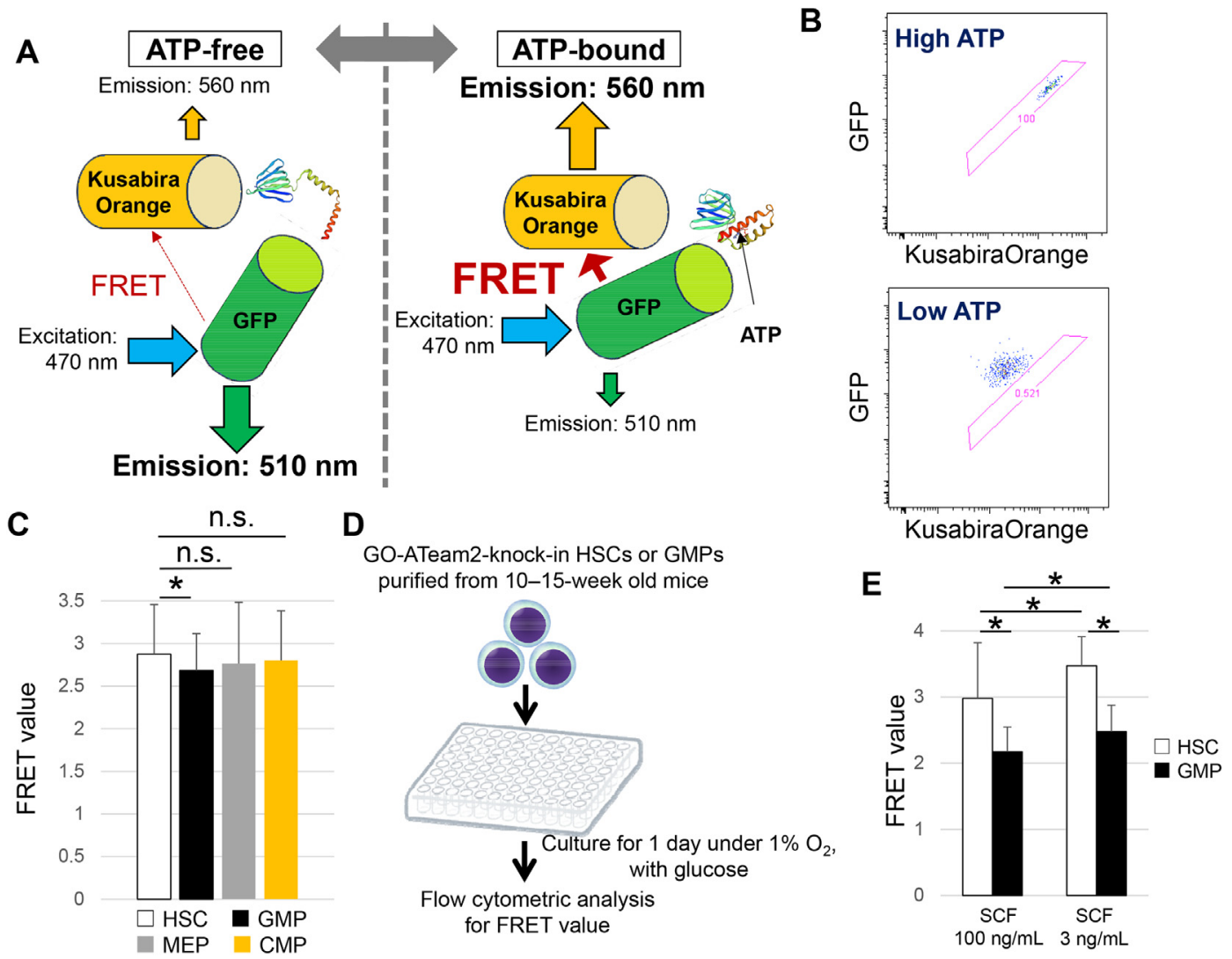

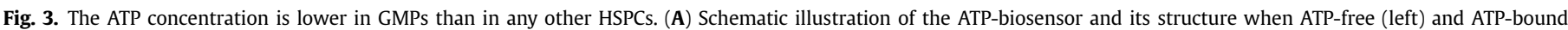

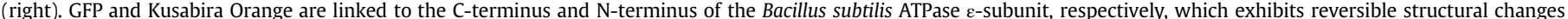

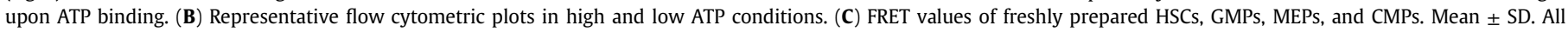

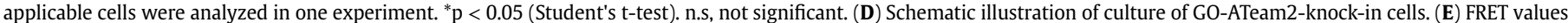

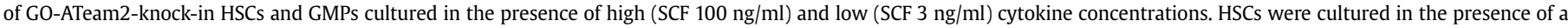

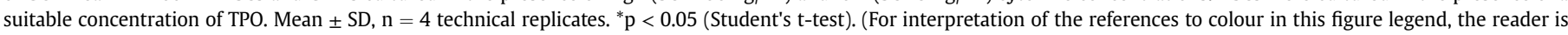
referred to the Web version of this article.)

concentrations (Fig. 4C and D). Under quiescent (low cytokine) conditions, HSCs flexibly became glucose-independent upon culture in glucose-free medium (Fig. 1). This metabolic plasticity of HSCs was lost in proliferative conditions and upon differentiation into HPCs (Fig. 1B and C). Dependency on glucose, which is a major source of ATP, is likely to reflect the ATP demand in each HSPC fraction. Consistently, ATP consumption was high in HPCs and proliferating HSCs, but low in quiescent HSCs. Experiments using a flux analyzer and transcriptome data revealed that ATP production was low in LSK cells (Fig. 2). By contrast, ATP production was higher in differentiated HPCs, such as GMPs and MEPs (Fig. 2A-E). This is consistent with previous reports that glycolytic ATP production is increased in parallel with ATP production via OXPHOS in differentiated cells [19]. In contrast with HPCs with a myeloid potential, CLPs exhibited low glycolytic and OXPHOS activities as well as low expression of glycolytic genes, despite their proliferating status (Fig. 2). GMPs displayed high glycolytic and OXPHOS activities as well as high expression of glycolytic and OXPHOS-related genes, while MEPs exhibited low glycolytic activity, low expression of glycolytic genes, and high expression of OXPHOS-related genes (Fig. 2F and G). The difference in glycolytic activity between GMPs and MEPs has also been reported in human cells [20]. These results suggest that the preference and activity of metabolic pathways for ATP production are not simply explained by the cell cycle status, but also by unique cellular characteristics at each differentiation stage. Thus, the combination of the differentiation stage and cell cycle status determines the ATP production potential of HSPCs. Among glycolytic enzymes, expression of Ldha and Pgam1 was higher in GMPs than in HSCs (Fig. 2F and G). The Ldha enzyme effectively converts pyruvate into lactate, regenerates $\mathrm{NAD}^{+}$necessary for glycolysis, and is required for maintenance of HSPCs [10]. Expression of Ldhb was higher in HSCs than in GMPs. Given that expression of Ldha was higher than that of Ldhb in both HSCs and GMPs, Ldha may define the metabolic characteristics of both cell types.

Experiments using GO-ATeam2-knock-in mice demonstrated that the ATP concentration was lower in GMPs than in other HSPCs. This can be explained by feedback regulation of ATP production in mitochondria. In general, mitochondrial ATP production is tightly coupled with the cellular ATP concentration. A low ATP concentration maintains high OXPHOS activity. Therefore, the equilibrium value of the cellular ATP concentration is determined by the ATP consumption rate in cells. As shown in this study, GMPs are ATPdemanding cells and thus the equilibrium value of the ATP concentration is lower in these cells than in less ATP-demanding cells, including HSCs. GMPs, MEPs, and CMPs used in this study are reportedly heterogeneous and GMPs can be contaminated by lymphoid committed progenitors [21]. Although our GMP culture efficiently generated $\mathrm{Gr}-1^{+}$or $\mathrm{Mac}-1^{+}$cells, further subfractionation of GMPs might clarify the additional metabolic heterogeneity among progenitors.

This study dissected alterations in metabolism of ATP from glucose in HSPC fractions according to the cell cycle status and 
A

HSC (with glucose)

B

\section{HSC (without glucose)}
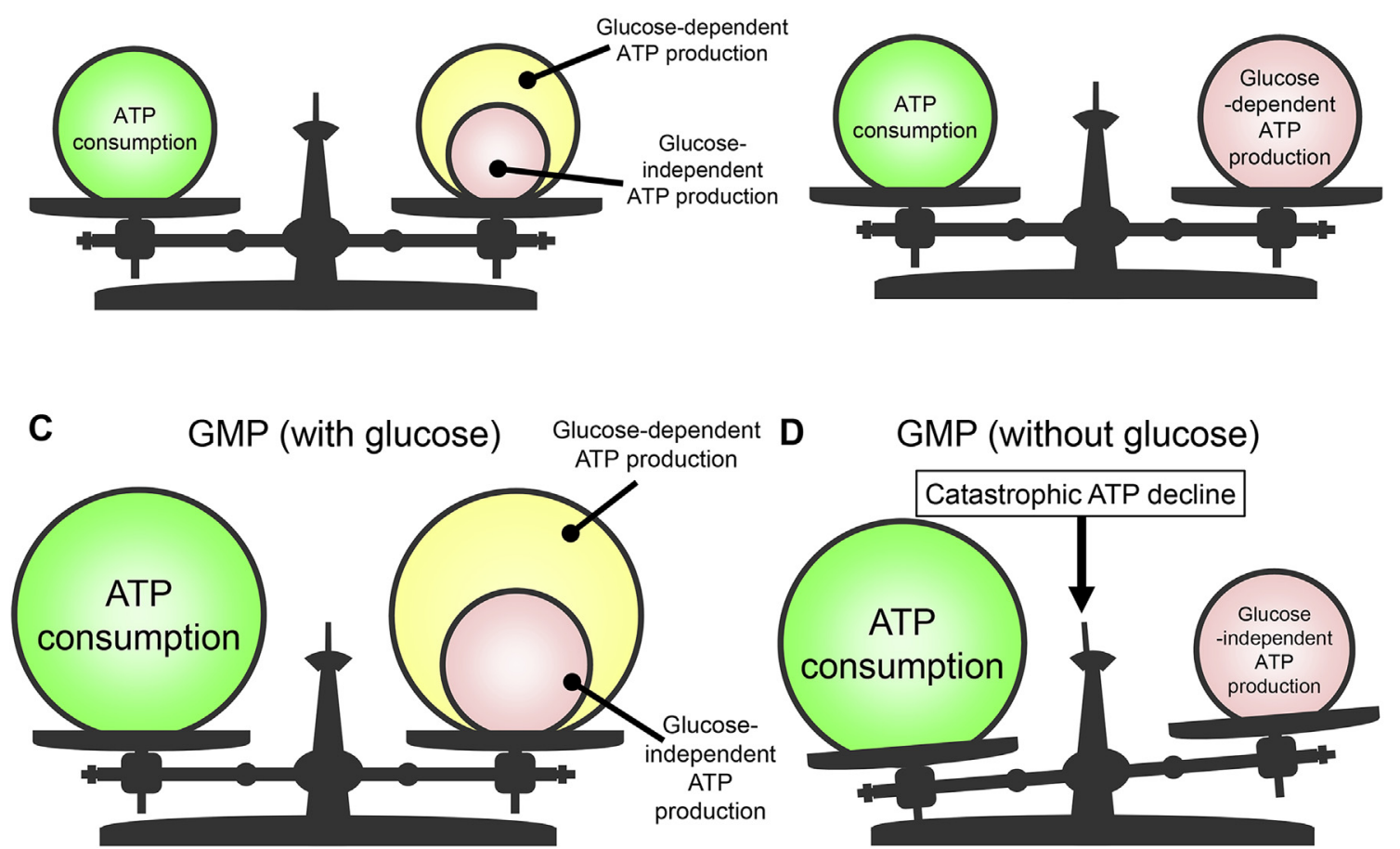

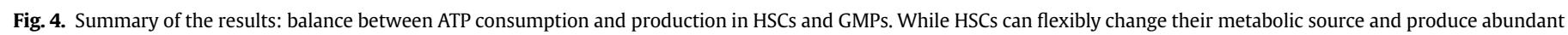

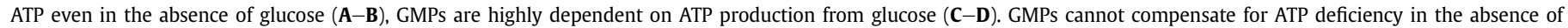
glucose, and consequently consumption and production of ATP become unbalanced.

differentiation stage. The next step to fully understand metabolic regulation in HSPCs is to elucidate how other energy sources, including lactic acid, amino acids, and fatty acids, impact cell cycledependent and differentiation-specific metabolic changes. Another intriguing area of further research is how HSCs lose their metabolic plasticity upon proliferation and differentiation.

\section{Conflicts of interest}

The authors declare no conflict of interest.

\section{Author contributions}

S.W., H.K., and Y.S. performed the study and analyzed the data. M.Y. and S.O. provided scientific advice and materials. S.W., H.K., and K.T. wrote the manuscript. K.T. conceived the project and supervised the research.

\section{Acknowledgments}

We thank all members of the Takubo laboratory for indispensable support as well as M. Haraguchi and S. Tamaki for technical support and laboratory management. KT was supported in part by KAKENHI grants from MEXT/JSPS (26115005, 18H02845, 18K19570, 26115001, and 15K21751), grants from the National Center for Global Health and Medicine (26-001 and 29-2007), AMED grants (JP18gm0710010, JP18bm0704011, and JP18ae0201014), and grants from the Takeda Science Foundation, the Kanzawa Medical Research Foundation, and the Ono Medical Research Foundation. HK was supported in part by a KAKENHI grant (17K16200), a grant from the National Center for Global Health and Medicine (29-1015), and grants from the Uehara Memorial Foundation and the Kanae Foundation for the Promotion of Medical Science. MY was supported in part by a PRESTO grant (JPMJPR14MF).

\section{Transparency document}

Transparency document related to this article can be found online at https://doi.org/10.1016/j.bbrc.2019.04.123.

\section{References}

[1] S.H. Orkin, L.I. Zon, Hematopoiesis: an evolving paradigm for stem cell biology, Cell 132 (2008) [631]-[644], https://doi.org/10.1016/j.cell.2008.01.025. S0092-8674(08)00125-6 [pii].

[2] W.M. Yu, X. Liu, J. Shen, O. Jovanovic, E.E. Pohl, S.L. Gerson, T. Finkel, H.E. Broxmeyer, C.K. Qu, Metabolic regulation by the mitochondrial phosphatase PTPMT1 is required for hematopoietic stem cell differentiation, Cell Stem Cell 12 (2013) 62-74, https://doi.org/10.1016/j.stem.2012.11.022.

[3] M. Maryanovich, Y. Zaltsman, A. Ruggiero, A. Goldman, L. Shachnai, S.L. Zaidman, Z. Porat, K. Golan, T. Lapidot, A. Gross, An MTCH2 pathway repressing mitochondria metabolism regulates haematopoietic stem cell fate, Nat. Commun. 6 (2015) 7901, https://doi.org/10.1038/ncomms8901.

[4] N.S. Chandel, H. Jasper, T.T. Ho, E. Passegué, Metabolic regulation of stem cell function in tissue homeostasis and organismal ageing, Nat. Cell Biol. 18 (2016) 823-832, https://doi.org/10.1038/ncb3385.

[5] D. Karigane, H. Kobayashi, T. Morikawa, Y. Ootomo, M. Sakai, G. Nagamatsu, Y. Kubota, N. Goda, M. Matsumoto, E.K. Nishimura, T. Soga, K. Otsu, M. Suematsu, S. Okamoto, T. Suda, K. Takubo, p38 $\alpha$ activates purine metabolism to initiate hematopoietic stem/progenitor cell cycling in response to stress, Cell Stem Cell 19 (2016) 192-204, https://doi.org/10.1016/ j.stem.2016.05.013.

[6] T. Schirmer, P.R. Evans, Structural basis of the allosteric behaviour of phosphofructokinase, Nature 343 (1990) 140-145, https://doi.org/10.1038/ $343140 \mathrm{a} 0$.

[7] R.M. Denton, P.J. Randle, B.J. Bridges, R.H. Cooper, A.L. Kerbey, H.T. Pask, D.L. Severson, D. Stansbie, S. Whitehouse, Regulation of mammalian pyruvate dehydrogenase, Mol. Cell. Biochem. 9 (1975) 27-53.

[8] R.A. Harris, J.W. Hawes, K.M. Popov, Y. Zhao, Y. Shimomura, J. Sato, J. Jaskiewicz, T.D. Hurley, Studies on the regulation of the mitochondrial alpha-ketoacid dehydrogenase complexes and their kinases, Adv. Enzym. Regul. 37 (1997) 271-293.

[9] M.J. Kiel, O.H. Yilmaz, T. Iwashita, C. Terhorst, S.J. Morrison, SLAM family 
receptors distinguish hematopoietic stem and progenitor cells and reveal endothelial niches for stem cells, Cell 121 (2005) 1109-1121, https://doi.org/ 10.1016/j.cell.2005.05.026. S0092-8674(05)00540-4 [pii].

[10] M. Kondo, I.L. Weissman, K. Akashi, Identification of clonogenic common lymphoid progenitors in mouse bone marrow, Cell 91 (1997) 661-672.

[11] K. Akashi, D. Traver, T. Miyamoto, I.L. Weissman, A clonogenic common myeloid progenitor that gives rise to all myeloid lineages, Nature 404 (2000) 193-197, https://doi.org/10.1038/35004599.

[12] H. Kobayashi, T. Morikawa, A. Okinaga, F. Hamano, T. Hashidate-Yoshida, S. Watanuki, D. Hishikawa, H. Shindou, F. Arai, Y. Kabe, M. Suematsu, T. Shimizu, K. Takubo, Environmental optimization enables maintenance of quiescent hematopoietic stem cells Ex Vivo, BioRxiv (2018) 475905, https:// doi.org/10.1101/475905.

[13] S. Comazzetto, M.M. Murphy, S. Berto, E. Jeffery, Z. Zhao, S.J. Morrison, Restricted hematopoietic progenitors and erythropoiesis require SCF from leptin Receptor+ niche cells in the bone marrow, Cell Stem Cell 24 (2019) 477-486, https://doi.org/10.1016/j.stem.2018.11.022, e476.

[14] K. Takubo, G. Nagamatsu, C.I. Kobayashi, A. Nakamura-Ishizu, H. Kobayashi, E. Ikeda, N. Goda, Y. Rahimi, R.S. Johnson, T. Soga, A. Hirao, M. Suematsu, T. Suda, Regulation of glycolysis by Pdk functions as a metabolic checkpoint for cell cycle quiescence in hematopoietic stem cells, Cell Stem Cell 12 (2013) 49-61, https://doi.org/10.1016/j.stem.2012.10.011.

[15] K. Takubo, N. Goda, W. Yamada, H. Iriuchishima, E. Ikeda, Y. Kubota, H. Shima, R.S. Johnson, A. Hirao, M. Suematsu, T. Suda, Regulation of the HIF-1 alpha level is essential for hematopoietic stem cells, Cell Stem Cell 7 (2010) [391]-
[402], https://doi.org/10.1016/j.stem.2010.06.020. S1934-5909(10)00344-9 [pii].

[16] F. Buttgereit, M.D. Brand, A hierarchy of ATP-consuming processes in mammalian cells, Biochem. J. 312 (Pt 1) (1995) 163-167.

[17] I. Beerman, J. Seita, M.A. Inlay, I.L. Weissman, D.J. Rossi, Quiescent hematopoietic stem cells accumulate DNA damage during aging that is repaired upon entry into cell cycle, Cell Stem Cell 15 (2014) 37-50, https://doi.org/10.1016/ j.stem.2014.04.016.

[18] M. Nakano, H. Imamura, T. Nagai, H. Noji, Ca2+ regulation of mitochondria ATP synthesis visualized at the single cell level, ACS Chem. Biol. 6 (7) (2011) 709-715, https://doi.org/10.1021/cb100313n.

[19] Y.H. Wang, W.J. Israelsen, D. Lee, V.W. Yu, N.T. Jeanson, C.B. Clish, L.C. Cantley, M.G. Vander Heiden, D.T. Scadden, Cell-state-specific metabolic dependency in hematopoiesis and leukemogenesis, Cell 158 (2014) 1309-1323, https:// doi.org/10.1016/j.cell.2014.07.048.

[20] L. Oburoglu, S. Tardito, V. Fritz, S.C. de Barros, P. Merida, M. Craveiro, J. Mamede, G. Cretenet, C. Mongellaz, X. An, D. Klysz, J. Touhami, M. BoyerClavel, J.L. Battini, V. Dardalhon, V.S. Zimmermann, N. Mohandas, E. Gottlieb, M. Sitbon, S. Kinet, N. Taylor, Glucose and glutamine metabolism regulate human hematopoietic stem cell lineage specification, Cell Stem Cell 15 (2014) 169-184, https://doi.org/10.1016/j.stem.2014.06.002.

[21] F. Paul, Y.A. Arkin, A. Giladi, D.A. Jaitin, E. Kenigsberg, H.,. Keren-Shaul E. David, Transcriptional heterogeneity and lineage commitment in myeloid progenitors, Cell 163 (7) (2015) 1663-1677, https://doi.org/10.1016/ j.cell.2015.12.046. 